\title{
A New Algorithms in Mixed Integer Programming Problems
}

\section{Basim A. Hassan}

basimabas39@gmial.com
Abbas Y. Al-Bayati

profabbasalbayati@yahoo.com

\section{College of Computer Sciences and Mathematics} University of Mosul

\section{Received on: 7/1/2007}

Accepted on: 16/4/2007

\section{ABSTRACT}

In this paper we have studied two new algorithms for solving mixed IPP. In the $1^{\text {st }}$ algorithm we have investigated a new short technique for searching to the efficient cut in the standard cutting plane procedure to obtain the same optimal solution by using a number of test problems in this field. In the $2^{\text {nd }}$ new algorithm we have put certain numerical conditions to obtain the global solution instead of the local solution by using cutting-plane and Al-Zobaidyi [1] algorithms. Our numerical results indicate that our new suggestions are efficient both numerically and theoretically compared with AlZobaidyi algorithm.

Keywords: Integer Programming Problems, Mixed Integer Programming, numerical results.

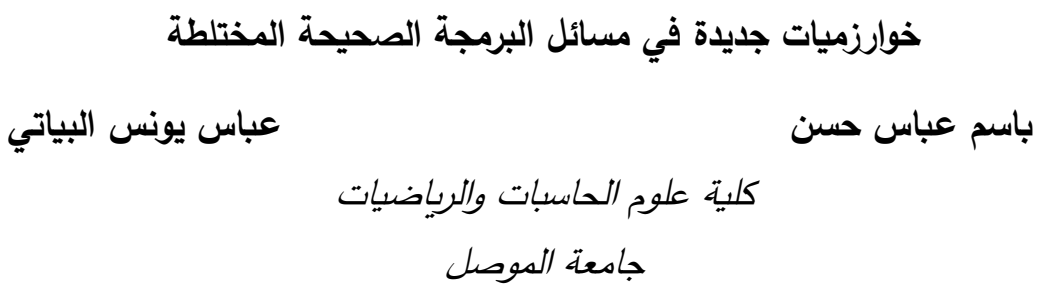

تاريخ قبول البحث: 2007/4/16

تاريخ استلام البحث: 2007/1/7

\section{الملخص}

تم التطرق في هذا البحث إلى خوارزميتين جديدتين لحل مسائل البرمجة الصحيحة المختلطة ـ وقد تم التقصي بوسيلة جديدة مختصرة في إيجاد القاطع الأكفأ في خوارزمية قطع المستويات القياسية مع الحصول على هلى فئل

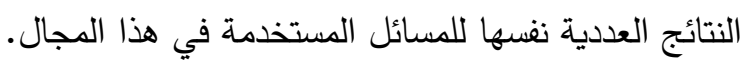

أما في الخوارزمية الثانية فقد تم وضع شروط عديدة للحصول على حل شامل بدلاً عن الحل الموضعي الذي يمكن الحصول عليه باستخدام خوارزميتي قطع المستويات والزبيدي [1]. النتائج العددية أثبتت كفاءة الخوارزميات

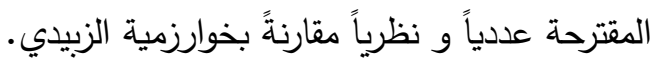
الكلمات المفتاحية : مسائل البرمجة الصحيحة, البرمجة الصحيحة المختلطة, النتائج العددية.

1 - 1 - 1 - 1 - 1

مسألة البرمجة الخطية الصحيحة هي مسألة برمجة خطية اعتيادية على أن تكون المتغيرات القرارية جميعها أو بعضها مقيدة وذات قيمة صحيحة أن طرق حل النماذج ذات الأعداد الصحيحة تعتمد بالأساس على مبدأين: المبدأ

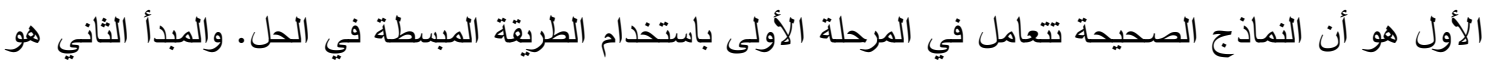
اعتماد النتائج النهائية التي تمثل الحل الأمثل كأساس للوصول للحل الأفضل الذي يجعل قيماً المتغيرات الأساسية قيم صحيحة (غير كسرية). ويتم بموجب ذلك اختيار قيود إضافية جديدة تشترك أو تؤمن قيم صحيحة للمتغيرات لتئل 
الأصلية في النموذج. يتركز هذا البحث أولا: على اختيارنا قيود إضافية جديدة وبإضافة هذه القيود الجديدة يتكون

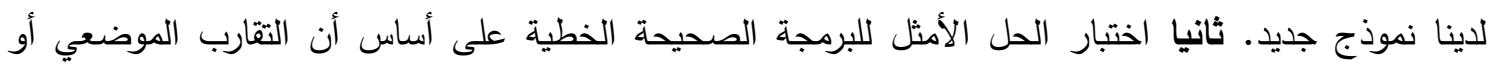

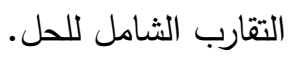

وهنا لا بد من ملاحظة أن إضافة القيود الجديدة تأتي بشكل متعاقب بحيث يتم إضافة قيد واحد إلى النموذج ثم تجري

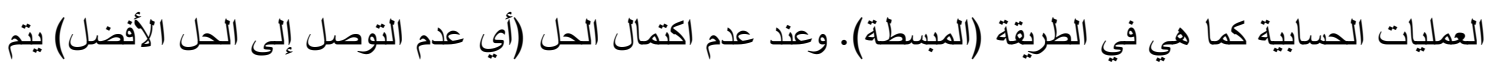

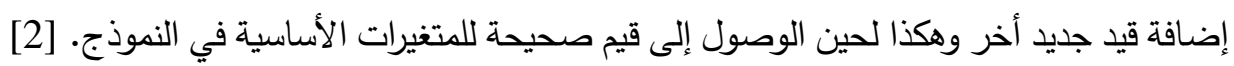

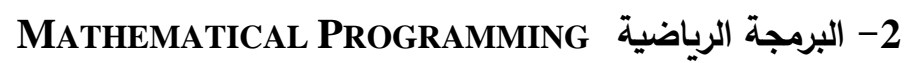

البرمجة الرياضية (M.P) هي أحدى الأساليب المستخدمة في صياغة المسائل الإدارية والاقتصادية وحلها

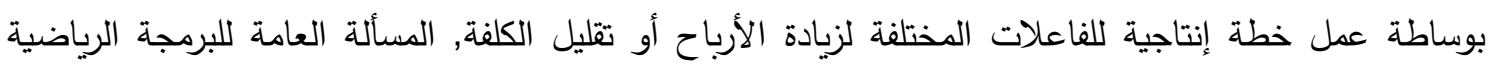

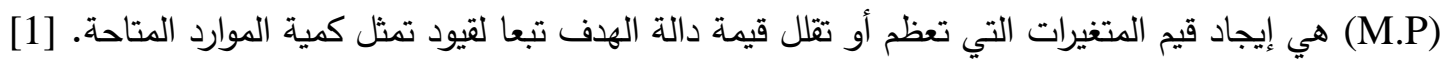
الصيغة العامة لمسألة البرمجة الرياضية هي على النحو الآتي : $z=c_{1} x_{1}+c_{2} x_{2}+\ldots \ldots \ldots \ldots+c_{n} x_{n}$

وتكون دالة الهدف هذه خاضعة لعدد من الهحدات أو القيود ( أي الثروط) والتي تتخذ الثكل الآتي: $a_{11} x_{1}+a_{12} x_{2}+$ $+a_{1 n} x_{n} \leq=\geq b_{1}$ $a_{21} x_{1}+a_{22} x_{2}+$ $+a_{2 n} x_{n} \leq=\geq b_{2}$

$a_{m 1} x_{1}+a_{m 2} x_{2}+$ $+a_{m n} x_{n} \leq=\geq b_{m}$

ومن الملاحظ أن المتغيرات $x_{j}$ هي متغيرات حقيقية (REAL VARIABLE) أي أن (x) (x) وان (CONSTANTS) ويستهدف من المسألة بهذه الصيغة أيجاد مجموعة من الأعداد الموجبة للمتغيرات المشمولة بالنظام بحيث تلبي

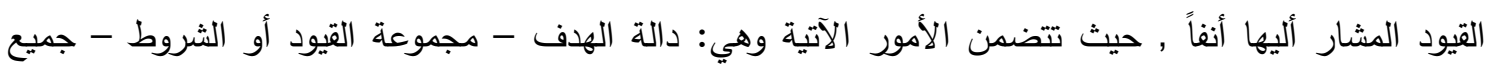
المتغيرات ذات قيم غير سالبة. [8]د

\section{3- مسألة البرمجة الخطية الصحيحة: INTEger Linear Programming ProbleM}

تتطلب أكثر التطبيقات العلمية لمسائل البرمجة الخطية (LP) حلا متمثلا بأعداد صحيحة فمثلا في مسائل الإنتاج فانه من غير الممكن أن يتمم إنتاج سيارة ونصف سيارة أو حقيبة جلدية وربع الحقيبة وعلى هذا الأساس ظهرت

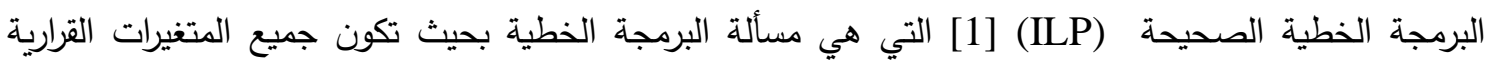
(الحقيقة) أو بعضها المقيدة لتكون ذات قيمة صحيحة , بدقة أكثر هي مسألة (تكبير أو تصغير ) دالة خطية (دالة الت التهية الهدف) ثابتة لمتغيرات ذات قيمة صحيحة وتحقق المعادلة (2)، بثكل عام مسألة البرمجة الصحيحة هي أكثر

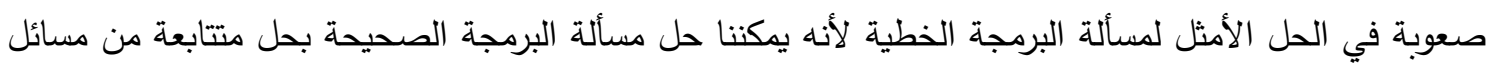
البرمجة الخطية. في مسألة البرمجة الصحيحة سوف نسمي أية قيمة صحيحة من المتغيرات التي تحقق (2) بالحل الموجب (FEASIBLE SOLUTION) ويمكننا أن نسمي مجموعة الحل الموجبة بالمنطقة الموجبة (FEASIBLE)

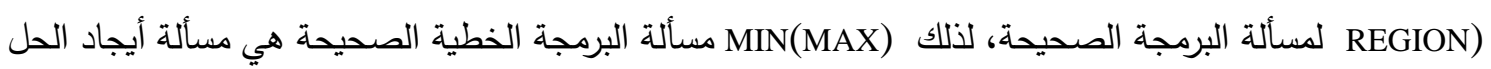


الموجب حيث تكون دالة الهدف اكبر (اصغر) أو تساوي قيمة كل الحلول الموجبة الأخرى الموجودة، فيسمى ذلك الحل بالحل الأمثل (OPTIMAL SOluTION) والذي هو هدفنا الرئيس. تحل مسألة البرمجة الخطية الصحيحة أولا بتجاهل شروط المتغيرات لأخذ قيم صحيحة، ثم تحل المسألة على إنها مسألة برمجة خطية لإيجاد الحل الحقيقي الأمثل، بعد ذلك نستخدم خوارزميات مسألة البرمجة الخطية

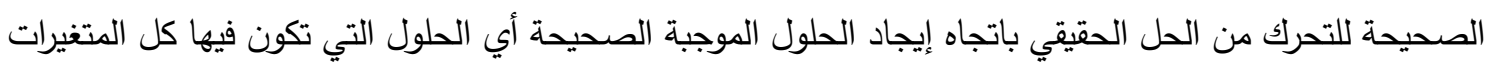
صحيحة، خوارزميات البرمجة الخطية الصحيحة تحاول أيجاد الحل الموجب الصحيح وبعد ذلك تبحث عن حل أخر الصراد أفضل. [4]

\section{4- طرائق لحل لمسألة البرمجة الخطية الصحيحة (ILP)}

هناك العديد من الطرائق التي تطورت لحل مسألة البرمجة الخطية الصحيحة (ILP) واغلب هذه الطرائق تقوم على أساس تجاهل قيد العدد الصحيح للتوصل إلى حل المسألة ومن ثم يتم معالجة القيم الكسرية للمتغيرات في

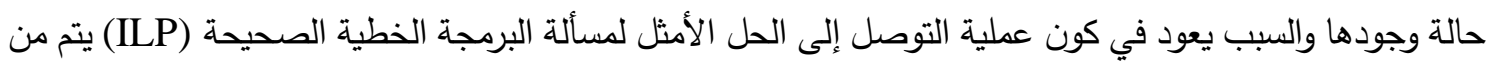
خلال الحل الأمثل لمسألة البرمجة الخطية (LP)، تقارب القيم المثلى للحل الأمثل للمسألتين وفي بعض التصلية الأحيان

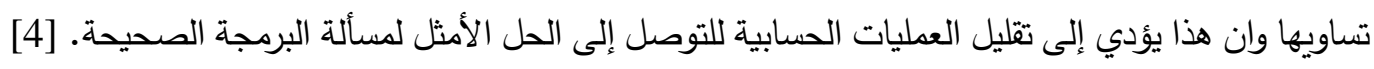

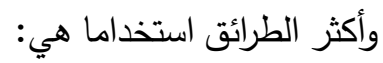

\section{THE CUTTING - PLANES METHOD 1-4 طريقة قطع المستويات}

هي طريقة فريدة من نوعها لحل مسألة البرمجة الخطية الصحيحة والتي نشرت عن طريق العالم [6]

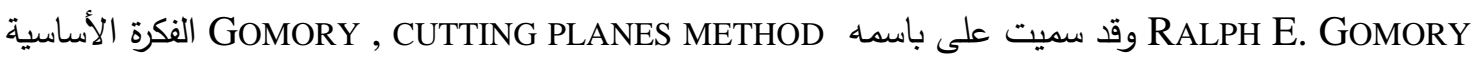
لطريقة قطع المستويات بسيطة لمسألة البرمجة الخطية الصحيحة، مسألة البرمجة الخطية هي بالضبط مسألة البرمجة

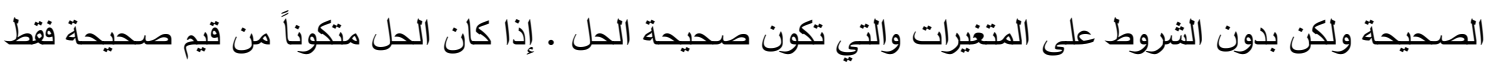

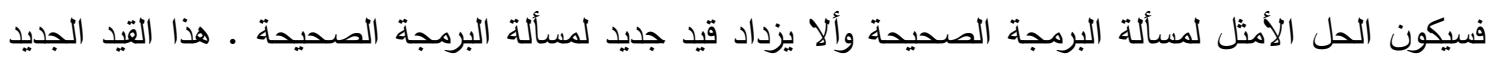

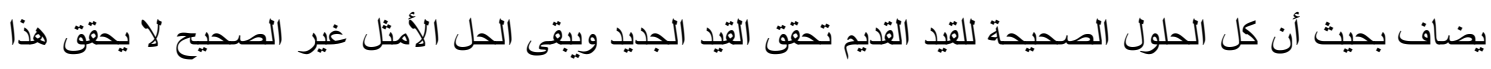

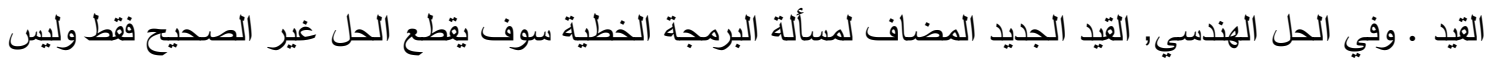
النقاط الصحيحة التي من الممكن أن تكون حلولا موجبة لمسألة البرمجة الصحيحة, نظريا (القطع) للحل غير الصحيح

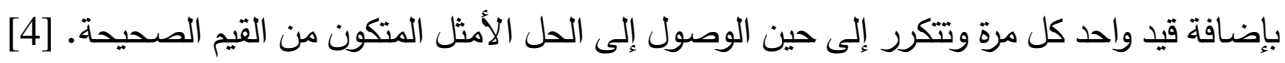
خوارزمية قطع المستويات: الخطوة 1: (الحل الابتدائي) ابدأ بحل المسألة المعطاة في (1) على أنها مسألة برمجة خطية بالطريقة الفردية Simplex Method

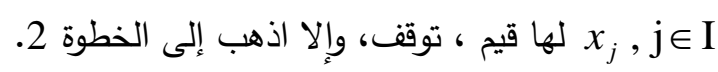

الخطوة 2: (اختيار القيد) اختر السطر من الجدول الأخير من حل مسألة البرمجة الخطية الذي يكون فيه المتغير الأساسي $x_{B i}$ ربما يساعد على تقليل عدد التكرارات والوقت المستغرق للتقارب) ومنه قم بتوليد قيد قطع المستويات. الخطوة 3: (توليد قيد قطع المستويات) افرض أن السطر المختار هو السطر $i$ ومعادلته هي: 


$$
\begin{aligned}
& x_{B i}+\sum_{j} a_{i j} x_{j}=b_{j}, \mathrm{j} \in \mathrm{I} \\
& x_{B i}+\sum_{j}\left(\left[a_{i j}\right]+f_{i j}\right) x_{j}=\left[b_{i}\right]+f_{i} \\
& x_{B i}+\sum_{j}\left[a_{i j}\right] x_{j}-\left[b_{i}\right]=f_{i}-\sum_{j} f_{i j} x_{j} \leq 0 \\
& f_{i}-\sum_{j} f_{i j} x_{j}+\delta=0
\end{aligned}
$$

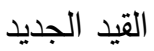

$$
\begin{aligned}
& \text { حيث } 0 \leq f_{i j} \prec 1, a_{i j} \text { لـ } \\
& \left.0 \leq f_{i} \prec 1, b_{i}\right\rfloor f_{i} f_{i}=b_{i}-\left[b_{i}\right] \\
& \text { • متغير منحل جديد ممكن وصحيح }
\end{aligned}
$$

الخطوة 4: (اختبار القاطع الأكفأ) نختبر القواطع التي نحصل عليها من الخطوة (3) لنحصل على القاطع الأكفأ

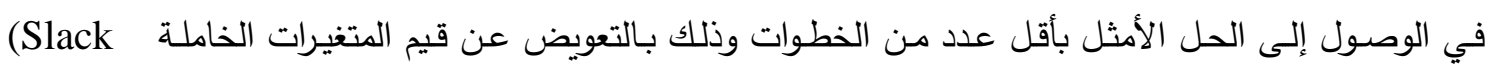

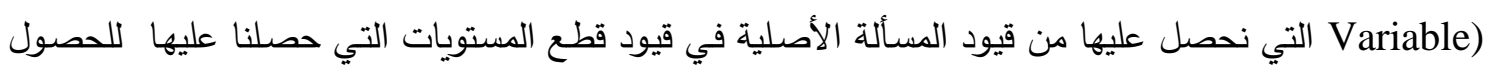

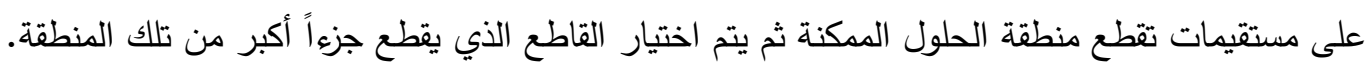
الخطوة 5: (إضافة القيد) قم بإضافة القيد (4) للجدول الأخير من حل الطريقة الفردية وقم بالحل على أنها مسألة البرمجة الخطية، إذا كانت جميع المتغيرات

\section{4-2- خوارزمية البرمجة الصحيحة (الزببياي)}

فلسفة طريقة البرمجة الصحيحة (الزبيدي) تعتمد على اختبارين اثثين الاختبار الأول يتمثل باختبار دالة

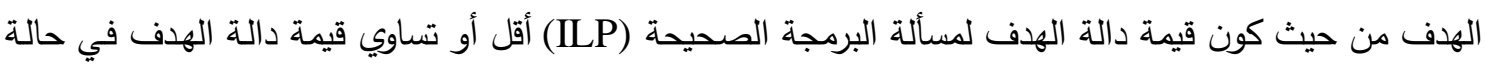
التعظيم أو أكبر أو تساوي قيمة دالة الهدف في حالة التقليل لمسألة البرمجة الخطية (LP) والاختبار الثاني يتمثل باختبار تحقق القيود [1]. وفي ما يأتي خطوات هذه الخوارزمية:

الخطوة 1: إيجاد الحل الأمثل للمسألة بدون الأخذ بنظر الاعتبار قيد العدد الصحيح

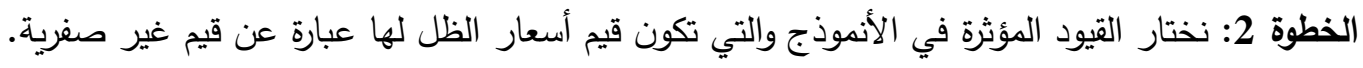

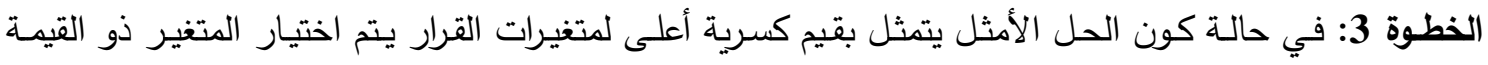

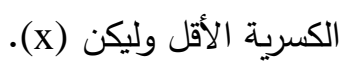

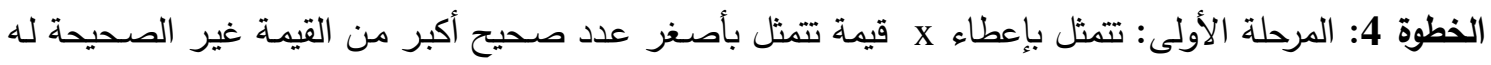
مع إعطاء بقية المتغيرات ذات القيمة غير الصحيحة قيم تتمثل بأكبر عدد صحيح أقل من القيم غير الصحيحة

الخطوة 5: حساب قيمة Z للمرحلة الأولى بتعويض القيم المختارة في الخطوة 4 بدالة الهدف. الخطوة 6: حساب

تساوي الصفر في حالة التقليل وعكس ذلك نتوقف وننتقل إلى المرحلة الثانية .

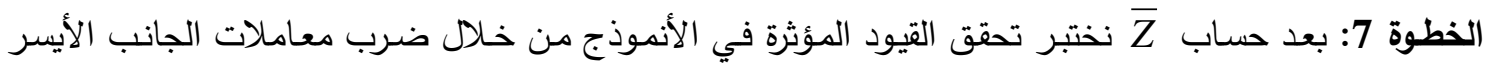
للقيود في الزيادة أو النقصان لقيم المتغيرات الناتجة من الخطوة 4. 
الخطوة 8: في حالة كون إثارة القيد أصغر أو يساوي فإن القيم المحسوبة في الخطوة 7 يجب أن تكون أقل أو

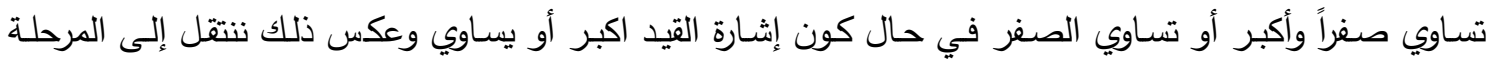

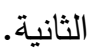
الخطوة 9: نختار القيد ذا القيمة غير الصفرية الأعلى الناتجة من الخطوة 7 في حال كون إشارة القيد أصغر أو

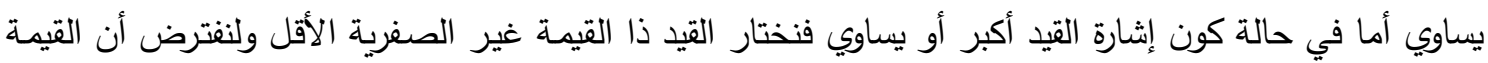
هي (k) . (1) الخطوة 10: حساب قيمة Q للقيد والتي تمثل معاملات الجانب الأيسر للقيد الأقل من أو تساوي | k| في حال كون إثارة القيد أصغر أو يساوي وأقل من أو تساوي k في حالة كون إثارة القيد أكبر أو يساوي وكذلك الاختلاف

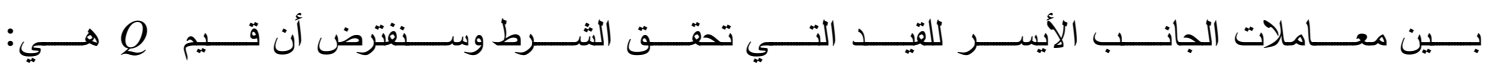
$Q=\left(a_{11}, a_{12}-a_{11}\right)$

الخطوة 11: في حال عدم وجود قيم لـ Q فإن حل المرحلة الأولى يمثل الحل الأمثل . الخطوة 12: حساب قيم 11: في حال علم وجود في لتي تمثل معاملات دالة الهدف بحيث أن :

$$
\begin{aligned}
& \bar{Q}=\left(c_{1}, c_{2}-c_{1}\right) \quad \text { إثارة القيد أصغر أو يساوي ولئ } \\
& \bar{Q}=\left(-c_{1}, c_{1}-c_{2}\right) \quad \text { إثارة القيد أكبر أو يساوي }
\end{aligned}
$$

الخطوة 13 : اختبار قيم $\bar{Q}$ الأكبر من الصفر والتي تكون اصغر أو تساوي $\bar{Z}$ في حالة التعظيم ومن ثم اختيار القيمة الأعلى منها أما في حالة التقليل فيتم اختيار قيم وعكس ذلك فإن حل المرحلة الأولى يمثل الحل الأمثل.

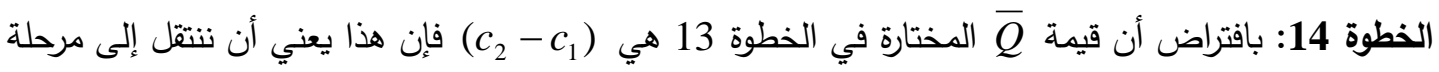
لاحقة تمثل زيادة قيمة المتغير $x_{2}$ ونقصان قيمة المتغير $x_{1}$ وحدة واحدة مع ثبات قيم المتغيرات الأخرى. الخطوة 15: يعاد حساب الخطوات السابقة ابتداءاً من الخطوة 4.

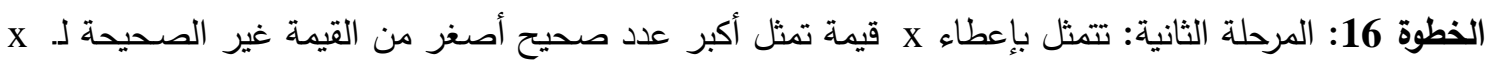
مع إعطاء بقية المتغيرات قيماً تتمثل بأصغر عدد صحيح أكبر من القيم غير الصحيحة لها ويعاد حساب الخطوات

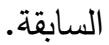

الخطوة 17: في حال كون المرحلة الأولى والثانية لا تمثل الحل الأمثل يتم إعطاء قيم لـ م المتغيرات تتمثل بأكبر عدد صحيح أقل من قيمها غير الصحيحة.

$$
\text { ويعاد حساب الخطوات السابقة. }
$$

5- 5وارزميات البرمجة الصحيحة المختطة الجديدة

\section{New Mixed Integer Linear Programming}

طريقة البرمجة الصحيحة المختلة تتطلب لصياغتها إجراء بعض التعديل في المسألة أو (الخوارزمية) كإضافة بعض الثروط الدالية أو استخدام بعض المتغيرات الإضافية [6] نود الإشارة إلى أن البرمجة الصحيحة

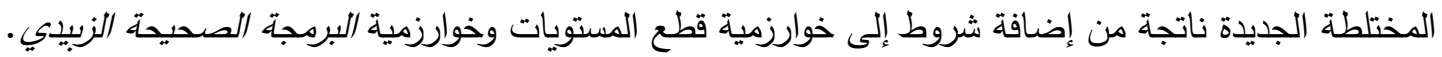


هي تقنية ناجحة جدا في حل مجموعة واسعة من مسائل البرمجة الصحيحة وهي توفير ضمانة الوصول

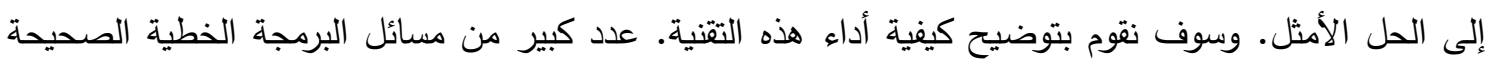

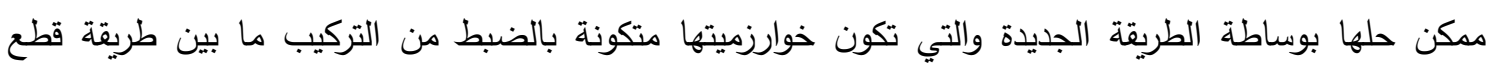

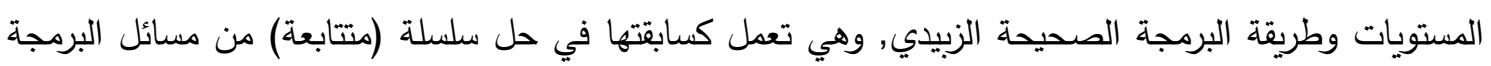

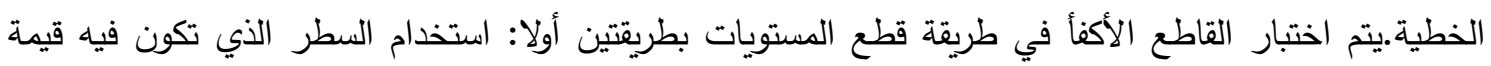
ذلك المتغير لها اكبر جزء كسري (من الحل الأمثل) ومنه نقوم بتوليد قيد قطع المستويات. ثانيا عن طريق الحل الهندسي حيث أن القيد الجديد المضاف لمسالة البرمجة الخطية سوف (يقطع) الحل غير الصحيحة فقط وليس

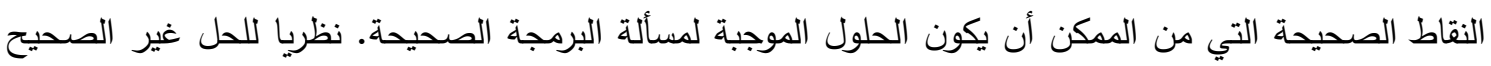
بإضافة قيد واحد كل مرة وتتكرر إلى حين الحصول على الأمثل المتكون من القيم الصحيحة. الطريقة الجديدة

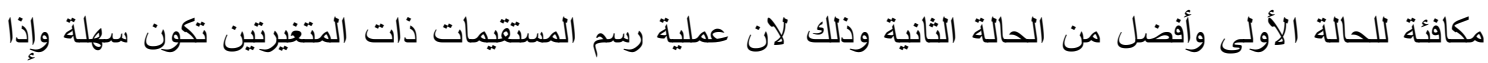
كان أكثر من المتغيرتين فيكون من صعب رسم المستقيمات, وقد يكون غير دقيق وهذا يؤدي إلى تقارب بطيء وربما لا تؤدي إلى الحل الأمثل.

الخوارزمية الأولى للبرمجة الصحيحة المختلة الجديدة:

الخطوة 1: (الحل الابتدائي) ابدأ بحل المسألة المعطاة في (1) على أنها مسألة البرمجة الخطية بالطريقة الفردية Simplex Method

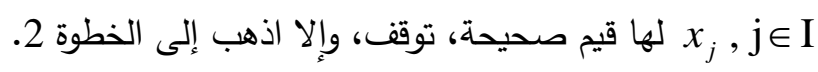

الخطوة 2: (اختيار القيد) اختر السطر من الجدول الأخير من حل مسألة البرمجة الخطية الذي يكون فيه المتغير

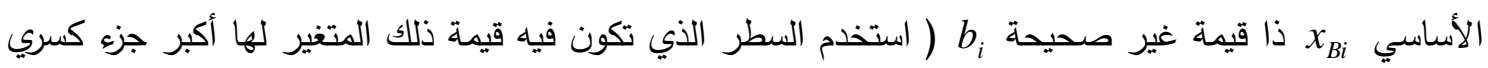
ربما يساعد على تقليل عدد التكرارات والوقت المستغرق للتقارب) ومنه قم بتوليد قيد قطع المستويات. الخطوة 3: (توليد قيد قطع المستويات) افرض أن السطر المختار هو السطر i $i$ ومعادلته هي فإن $x_{B i}+\sum_{j} a_{i j} x_{j}=b_{j}, \mathrm{j} \in \mathrm{I}$

$x_{B i}+\sum_{j}\left(\left[a_{i j}\right]+f_{i j}\right) x_{j}=\left[b_{i}\right]+f_{i}$

$x_{B i}+\sum_{j}\left[a_{i j}\right] x_{j}-\left[b_{i}\right]=f_{i}-\sum_{j} f_{i j} x_{j} \leq 0$

$f_{i}-\sum_{j} f_{i j} x_{j}+\delta=0$

القيد الجديد

$$
\begin{aligned}
& \text { حيث } 0 \leq f_{i j} \prec 1, a_{i j} \text { لـ } \\
& \left.0 \leq f_{i} \prec 1, b_{i}\right\rfloor f_{i}=b_{i}-\left[b_{i}\right]
\end{aligned}
$$

ס متغير منحل جديد ممكن وصحيح.

الخطوة 4: بعد توليد قيود قطع المستويات اختبر تحقق القيود المؤثرة في الأنموذج من خلال ضرب معن معاملات

الجانب الأيسر للقيود في الزيادة أو النقصان لقيم المتغيرات الناتجة من الخطوة 2. الخطوة 5: في حالة كون إثارة القيد أصغر أو يساوي فإن القيم المحسوبة في الخطوة 4 يجب التباني أن تكون أقل أو أو تساوي صفر وأكبر أو تساوي الصفر في حال كون إشارة القيد اكبر أو يساوي. 
الخطوة 6: اختر القيد ذا القيمة غير الصفرية الأعلى الناتجة من الخطوة 4 في حال كون إثارة القيد أصغر أو يساوي أما في حالة كون إثارة القيد أكبر أو يساوي فاختر القيد ذا القيمة غير الصنيه الصفرية الأقل. الخطوة 7: (إضافة القيد) قم بإضافة القيد الذي حصلنا عليه من الخطوة 6 للجدول الأخير من حل الطريقة الفردية وقم بالحل على أنها مسألة البرمجة الخطية، إذا كانت جميع المتغيرات

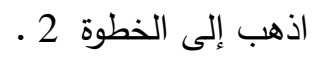
من الخطوات (4) إلى (7) يتم اختيار القاطع الجديد للتكرار التالي ولا يعتمد على طريقتي اختبار القاطع الأكفأ المستخدم في خوارزمية قطع المستوي.

2-5- طريقة ثانية للبرمجة الصحيحة المختلةة الجديدة (NEW 2): ندرج فيما يأتي الخطوات التكرارية المطلوبة في الخوارزمية المقترحة الجديدة على النحو الأتي: الخوارزمية الثانية للبرمجة الصحيحة المختلطة الجديدة: الخطوة 1: إيجاد الحل الأمثل للمسألة بدون الأخذ بعين الاعتبار قيد العدد الصحيح الخطوة 2: نختار القيود المؤثرة في الأنموذج والتي تكون قيم أسعار الظل لها عبارة عن قيم غير الإنير صغرية. الخطوة 3: في حالة كون الحل الأمثل يتمثل بقيم كسرية أعلى لمتغيرات القرار يتم اختيار المتغير ذي القيمـة الكسرية الأقل وليكن (x). (x). الخطوة 4: المرحلة الأولى: تتمثل بإعطاء X Xيمة تتمثل بأصغر عدد صحيح أكبر من القيمة غير الصحيحة له مع إعطاء بقية المتغيرات ذات القيمة غير الصحيحة قيماً تتمثل بأكبر عدد صحيح أقل من القيم غير الصير الصحيحة

الخطوة 5: حساب قيمة Z للمرحلة الأولى بتعويض القيم المختارة في الخطوة 4 بدالة الهدف. الخطوة 6: حساب أو تساوي الصفر في حالة التقليل وعكس ذلك نتوقف وننتقل إلى المرحلة الثانية.

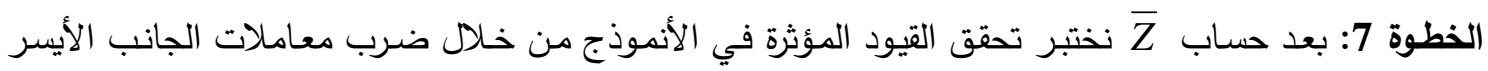

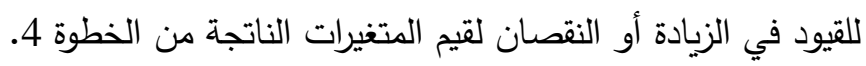
الخطوة 8: في حالة كون إثارة القيد أصغر أو يساوي فإن القيم المحسوبة في الخطوة 7 يجب أنبان أن تكون أقل أو

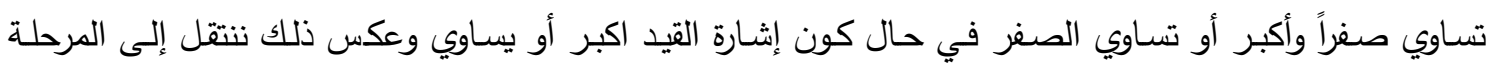
الثانية. الخطوة 9: نختار القيد ذا القيمة غير الصفرية الأعلى الناتجة من الخطوة 7 في حال كون إشارة القيد أصغر أو

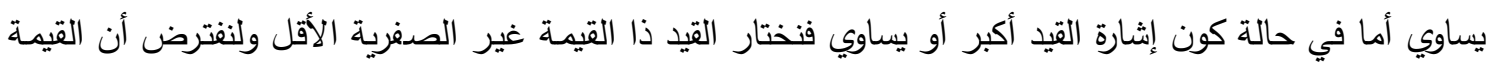
هي (k). الخطوة 10: حساب قيمة Q للقيد والتي تمثل معاملات الجانب الأيسر للقيد الأقل من أو تساوي | k| في حال كون إثارة القيد أصغر أو يساوي وأقل من أو تساوي k في حالة كون إشارة القيد أكبر أو يساوي وكذلك الاختلاف

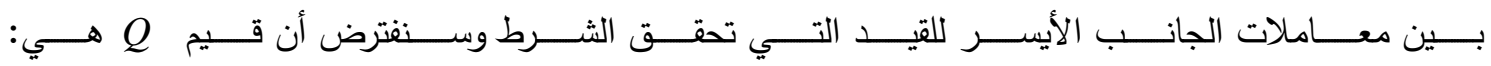
$Q=\left(a_{11}, a_{12}-a_{11}\right)$ الخطوة 11: في حال عدم وجود قيم لـ Q فإن حل المرحلة الأولى يمثل الحل الأمثل 
الخطوة 12: حساب قيم

$$
\begin{array}{lr}
\bar{Q} & =\left(c_{1}, c_{2}-c_{1}\right) \\
\bar{Q} & =\left(-c_{1}, c_{1}-c_{2}\right)
\end{array}
$$

الخطوة 13: اختبار قيم $\bar{Q}$ الأكبر من الصفر والتي تكون اصغر أو تساوي

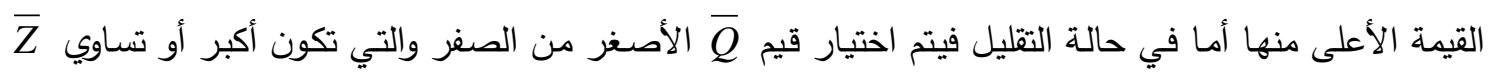
وعكس ذلك فإن حل المرحلة الأولى يمثل الحل الأمثل.

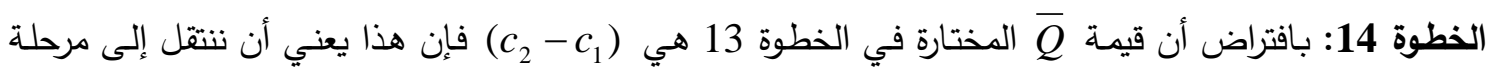
لاحقة تمثل زيادة قيمة المتغير x 2 ونقصان قيمة المتغير $x_{1}$ وحدة واحدة مع ثبات قيم المتغيرات الأخرى. الخطوة 15: يعاد حساب الخطوات السابقة ابتداءاً من الخطوة 4. الخطوة 16: المرحلة الثانية: تتمثل بإعطاء x قيمة تمثل أكبر عدد صحيح أصغر من القيمة غير الصحيحة لـ

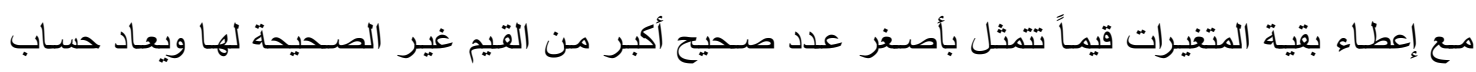
الخطوات السابقة. الخطوة 17: في حال كون المرحلة الأولى والثانية لا تمثل الحل الأمثل فيتم إعطاء قيم لـ x والمتغيرات تتمثل بأكبر عدد صحيح أقل من قيمها غير الصحيحة. ويعاد حساب الخطوات السابقة.

الخطوة 18: المرحلة الثالثة : تتمثل بإعطاء $n$,......., عدا $i=2$ وهكذا نستمر • ويتم حساب قيم أخرى لـ بتقسيم قيمة Z الموجودة في دالة الهدف. الخطوة 19: حساب قيمة $Z_{i}$ للمرحلة الثالثة بتعويض القيم المختارة في الخطوة 18 بدالة الهذف. الخطوة 20: حساب 19 $\bar{Z}=Z-Z$ بحيث قيمة $\bar{Z}$ يجب أن تكون أكبر أو تساوي الصفر في حالة التعظيم وأقل أو تساوي الصفر في حالة التقليل وعكس ذلك يعد الحل الذي حصلنا عليه في المرحلة الأخيرة الحل الأمثل. الخطوة 21: بعد حساب $\bar{Z}$ نختبر تحقق القيود المؤثرة في الأنموذج من خلال ضرب معاملاتلات الجانب الأيسر

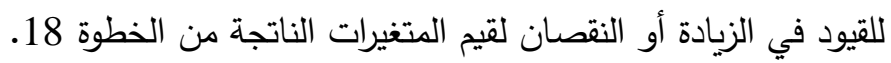
الخطوة 22: في حالة كون إثارة القيد أصغر أو يساوي فإن القيم المحسوبة في الخطوة 21 يجب أنبان أن تكون أقل أو أو تساوي صفراً وأكبر أو تساوي الصفر في حال كون إثـارة القيد اكبر أو يساوي, إذا تحققت القيود نقارن

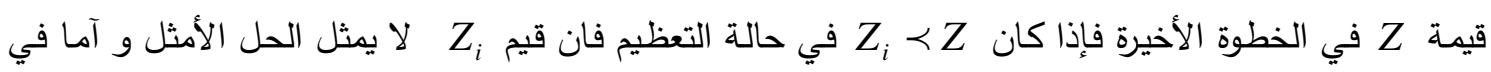
حالة التقليل يكون في هذه الخوارزمية من الخطوات (18)-(22) يتم الحصول على حل أمثل شامل دائماً في حين أن الخوارزمية الزببيدي

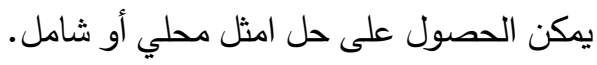


تم تطبيق الطرائق المقترحة الجديدة على العديد من الأمثلة من اجل بيان كفاءتها. وفي هذه الفقرة سوف نستعرض بعض هذه الأمثلة للتوضيح:

مثال (1): حل مسألة البرمجة الخطية الصحيحة التالية :

$$
\begin{array}{ll}
\operatorname{Max} Z= & 5 x_{1}+8 x_{2} \\
\text { s.t } \quad & x_{1}+x_{2} \leq 6 \\
& 5 x_{1}+9 x_{2} \leq 45 \\
& x_{1}, x_{2} \geq 0 \quad \text { and int egers }
\end{array}
$$

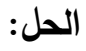

Simplex Method الطريقة الفردية

$$
\begin{array}{ll}
\text { MinU } & =-5 x_{1}-8 x_{2} \\
\text { s.t } \quad & x_{1}+x_{2}+\delta_{1}=6 \\
& 5 x_{1}+9 x_{2}+\delta_{2}=45 \\
& x_{1}, x_{2}, \delta_{1}, \delta_{2} \geq 0
\end{array}
$$

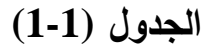

\begin{tabular}{|c|c|c|c|c|c|}
\hline Basic & $b_{i}$ & $x_{1}$ & $x_{2}$ & $\delta_{1}$ & $\delta_{2}$ \\
\hline$x_{1}$ & $9 / 4$ & 1 & 0 & $9 / 4$ & $-1 / 4$ \\
\hline$x_{2}$ & $15 / 4$ & 0 & 1 & $-5 / 4$ & $1 / 4$ \\
\hline$-U$ & $165 / 7$ & 0 & 0 & $5 / 4$ & $3 / 4$ \\
\hline
\end{tabular}

الحل الأمثل لمسألة البرمجة الخطية دون الأخذ بعين الاعتبار قيد العدد الصحيح هو:

$$
x_{1}=9 / 4, x_{2}=15 / 4 \quad, \quad Z=41 \frac{1}{4}
$$

استخدام طريقة الأولى للبرمجة الصحيحة المختلة الجديدة من الجدول (1-1) نحصل على:

$$
\begin{aligned}
-Z-\frac{5}{4} \delta_{1}-\frac{3}{4} \delta_{2} & =-41 \frac{1}{4} \\
x_{1}+\frac{9}{4} \delta_{1}-\frac{1}{4} \delta_{2} & =\frac{9}{4} \\
x_{2}-\frac{5}{4} \delta_{1}+\frac{1}{4} \delta_{2} & =\frac{15}{4} \\
x_{1}, x_{2}, \delta_{1}, \delta_{2} & \geq 0 \\
-Z-2 \delta_{1}-\delta_{2}+42 & =\frac{3}{4}-\frac{3}{4} \delta_{1}-\frac{1}{4} \delta_{2}
\end{aligned}
$$

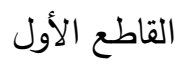




$$
\begin{aligned}
& x_{1}+2 \delta_{1}-\delta_{2}-2=\frac{1}{4}-\frac{1}{4} \delta_{1}-\frac{3}{4} \delta_{2} \\
& x_{2}-2 \delta_{1}-3=\frac{3}{4}-\frac{3}{4} \delta_{1}-\frac{1}{4} \delta_{2} \\
& \text { القاطع الثالث } \\
& \frac{3}{4}-\frac{3}{4} \delta_{1}-\frac{1}{4} \delta_{2} \leq 0 \\
& \text { القواطع } \\
& \frac{1}{4}-\frac{1}{4} \delta_{1}-\frac{3}{4} \delta_{2} \leq 0 \\
& \text { الأول=الثالث } \\
& \frac{3}{4}-\frac{3}{4} \delta_{1}-\frac{1}{4} \delta_{2}+\delta_{3}=0 \quad, \delta_{3} \geq 0 \\
& \frac{1}{4}-\frac{1}{4} \delta_{1}-\frac{3}{4} \delta_{2}+\delta_{4}=0 \quad, \delta_{4} \geq 0
\end{aligned}
$$

$\delta_{2}=45-5 x_{1}-9 x_{2}$

نعوض المعادلات (6) و (7) في القواطع الأول والثاني لإيجاد القواطع الأساسية في المسألة والتي هي بدلالمة المتغيرات في المسألة

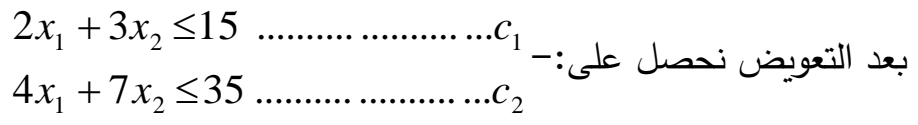

نختبر القاطع الأكفأ وذلك بتعويض مقدار الزيادة أو النقصان في قيم $\left(-\frac{1}{4}\right) 2+\left(-\frac{3}{4}\right) 3=-2 \frac{3}{4}$

$\left(-\frac{1}{4}\right) 4+\left(-\frac{3}{4}\right) 7=-6 \frac{1}{4}$

بما أن القاطع الأكفأ هو القاطع الأول، لذلك نستخدم القاطع الأول لحل المسألة

$\frac{3}{4}-\frac{3}{4} \delta_{1}-\frac{1}{4} \delta_{2} \leq 0$

$\frac{3}{4}-\frac{3}{4} \delta_{1}-\frac{1}{4} \delta_{2}+\delta_{3}=0, \delta_{3} \geq 0$

$-\frac{3}{4} \delta_{1}-\frac{1}{4} \delta_{2}+\delta_{3}=-\frac{3}{4}$ 
$\Downarrow$

الجدول (1-2)

\begin{tabular}{|c|c|c|c|c|c|c|}
\hline Basic & $b_{i}$ & $x_{1}$ & $x_{2}$ & $\delta_{1}$ & $\delta_{2}$ & $\delta_{3}$ \\
\hline$x_{1}$ & $9 / 4$ & 1 & 0 & $9 / 4$ & $-1 / 4$ & 0 \\
\hline$x_{2}$ & $15 / 4$ & 0 & 1 & $-5 / 4$ & $1 / 4$ & 0 \\
\hline$-U$ & $165 / 4$ & 0 & 0 & $5 / 4$ & $3 / 4$ & 0 \\
\hline$\delta_{3}$ & $-3 / 4$ & 0 & 0 & $-3 / 4$ & $-1 / 4$ & 1 \\
\hline
\end{tabular}

الجدول (3-1)

\begin{tabular}{|c|c|c|c|c|c|c|}
\hline Basic & $b_{i}$ & $x_{1}$ & $x_{2}$ & $\delta_{1}$ & $\delta_{2}$ & $\delta_{3}$ \\
\hline$x_{1}$ & 0 & 1 & 0 & 0 & -1 & 3 \\
\hline$x_{2}$ & 5 & 0 & 1 & 0 & $2 / 3$ & $-5 / 3$ \\
\hline$-U$ & 40 & 0 & 1 & 0 & $-1 / 3$ & $-5 / 3$ \\
\hline$\delta_{1}$ & 1 & 0 & 0 & 1 & $1 / 3$ & $-4 / 3$ \\
\hline
\end{tabular}

$$
x_{1}=0, x_{2}=5, \quad Z=40 \quad \quad \quad \text { إذا الجواب }
$$

استخدام طريقة ثانية للبرمجة الصحيحة المختلطة الجديدة الجدول (1-1) يمثل الحل الأمثل للمسالة دون الآخذ بعين الاعتبار قيد العدد الصحيح وهو

$$
x_{1}=2 \frac{1}{4} \quad, x_{2}=3 \frac{3}{4}, Z=41 \frac{1}{4}
$$

قيم أسعار الظل هي

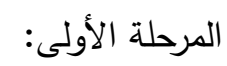
نختار المتغير $x_{2}$

$$
\begin{aligned}
& x_{1}=2, x_{2}=4, Z_{1}=42 \\
& \bar{Z}=Z-Z_{1}=41 \frac{1}{4}-42=-\frac{3}{4}
\end{aligned}
$$

بما أن القيمة سالبة ننتقل مباشرة إلى المرحلة الثانية. المرحلة الثانية:

$$
\begin{aligned}
& x_{1}=3, x_{2}=3, Z_{2}=39 \\
& \bar{Z}=Z-Z_{2}=41 \frac{1}{4}-39=2 \frac{1}{4}
\end{aligned}
$$
1) $\left(\frac{3}{4}\right) 1-\left(\frac{3}{4}\right) 1=0$
2) $\left(\frac{3}{4}\right) 5-\left(\frac{3}{4}\right) 9=-3$ 
بما أن القيم ( 3-,0 ) اصغر أو تساوي الصفر فهذا يعني تحقق القيود ومن القيم ( 3-,0 ) يتم اختبار القيد الثاني

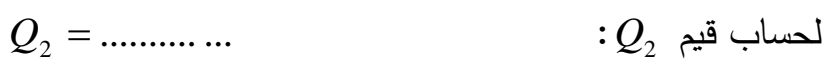$$
x_{1}=3, x_{2}=3, \mathrm{Z}=39
$$

الحل

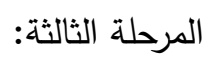

$x_{1}=0, x_{2}=5, Z_{3}=40$
$\bar{Z}=Z-Z_{3}=41 \frac{1}{4}-40=1 \frac{1}{4}$

1) $-1\left(\frac{9}{4}\right)+1\left(\frac{5}{4}\right)=-1$

2) $-5\left(\frac{9}{4}\right)+9\left(\frac{5}{4}\right)=0$

$Q_{1}=\left(a_{11}, a_{12}-a_{11}, a_{11}-a_{12}\right)$

بما أن القيم اقل أو تساوي صفر هذا يعني تحقق القيود

$\overline{Q_{1}}=$

المرحلة الرابعة: - م الم

$x_{1}=8, x_{2}=0, Z_{4}=40$

$\bar{Z}=Z-Z_{4}=41 \frac{1}{4}-40=1 \frac{1}{4}$

نختبر تحقق القيود:

1) $1\left(\frac{23}{4}\right)-1\left(\frac{15}{4}\right)=2$

2) $5\left(\frac{23}{4}\right)-9\left(\frac{15}{4}\right)=-\frac{30}{4}$

بما إن ليست جميع القيم اقل أو تساوي صفر هذا يعني لا تحقق القيود إذن النقطة لا تمثل حل ممكن للمسالة ـ إذا الحل الأمثل ل

المثال (2): حل مسألة البرمجة الخطية الصحيحة التالية:

$\operatorname{Min} Z=2 x_{1}-3 x_{2}-3 x_{3}$

$$
\begin{aligned}
& \text { s.t } \quad-x_{1}+x_{2}+3 x_{3} \leq 8 \\
& 3 x_{1}+2 x_{2}-x_{3} \leq 10 \\
& \\
& x_{1}, \quad x_{2}, \quad x_{3} \geq 0
\end{aligned}
$$

الحل:

Simplex Method الطريقة الفردية: 


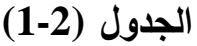

\begin{tabular}{|c|c|c|c|c|c|c|}
\hline Basic & $b_{i}$ & $x_{1}$ & $x_{2}$ & $x_{3}$ & $\delta_{1}$ & $\delta_{2}$ \\
\hline$x_{3}$ & $6 / 7$ & $-5 / 7$ & 0 & 1 & $2 / 7$ & $-1 / 7$ \\
\hline$x_{2}$ & $38 / 7$ & $8 / 7$ & 1 & 0 & $1 / 7$ & $3 / 7$ \\
\hline $\mathrm{Z}$ & $132 / 7$ & $23 / 7$ & 0 & 0 & $9 / 7$ & $6 / 7$ \\
\hline
\end{tabular}

الحل الأمثل لمسألة البرمجة الخطية دون الأخذ بعين الاعتبار قيد العدد الصحيح هو: $x_{3}=6 / 7, x_{2}=38 / 7 \quad, \quad Z=-132 / 7$

استخدام طريقة الأولى للبرمجة الصحيحة المختلطة الجديدة من الجدول (2 - 1) نحصل على :

$$
\begin{aligned}
& Z+\frac{9}{7} \delta_{1}+\frac{6}{7} \delta_{2}+\frac{23}{7} x_{1}=\frac{132}{7} \\
& x_{2}+\frac{1}{7} \delta_{1}+\frac{3}{7} \delta_{2}+\frac{8}{7} x_{1}=\frac{38}{7} \\
& x_{3}+\frac{2}{7} \delta_{1}-\frac{1}{7} \delta_{2}-\frac{5}{7} x_{1}=\frac{6}{7} \\
& x_{1}, x_{2}, x_{3}, \delta_{1}, \delta_{2} \geq 0 \\
& Z+3 x_{1}+\delta_{1}-18=\frac{6}{7}-\frac{2}{7} x_{1}-\frac{2}{7} \delta_{1}-\frac{6}{7} \delta_{2} \leq 0
\end{aligned}
$$

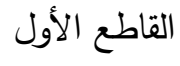

$$
\begin{aligned}
& x_{2}+x_{1}-5=\frac{3}{7}-\frac{1}{7} x_{1}-\frac{1}{7} \delta_{1}-\frac{3}{7} \delta_{2} \leq 0 \\
& x_{3}-x_{1}-\delta_{2}=\frac{6}{7}-\frac{2}{7} x_{1}-\frac{2}{7} \delta_{1}-\frac{6}{7} \delta_{2} \leq 0
\end{aligned}
$$

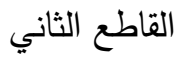

$$
\begin{aligned}
& \text { القاطع الثالث } \\
& \frac{6}{7}-\frac{2}{7} x_{1}-\frac{2}{7} \delta_{1}-\frac{6}{7} \delta_{2} \leq 0 \\
& \frac{3}{7}-\frac{1}{7} x_{1}-\frac{1}{7} \delta_{1}-\frac{3}{7} \delta_{2} \leq 0
\end{aligned}
$$

القواطع

اختبار القاطع الأكفأ من ضمن القواطع المستخرجة من المسألة:

$$
\begin{array}{lll}
\frac{6}{7}-\frac{2}{7} x_{1}-\frac{2}{7} \delta_{1}-\frac{6}{7} \delta_{2}+\delta_{3}=0 & , \delta_{3} \geq 0 & \text { القاطع الأول القبار الثاني }
\end{array}
$$

من المسألة الأصلية نستخرج قيم

$\delta_{1}=8+x_{1}-x_{2}-3 x$

$\delta_{2}=10-3 x_{1}-2 x_{2}+x_{3}$

نعوض المعادلات (8),(9) في القواطع الأولى والثانية لإيجاد القواطع الأساسية في المسألة والتي هي بدلالة المتغيرات في المسألة 
$2 x_{1}+2 x_{2} \leq 10$

$x_{1}+x_{2} \leq-5$

$2(0)+2\left(-\frac{3}{7}\right)+0=-\frac{6}{7}$

$1(0)+1\left(-\frac{3}{7}\right)+0=-\frac{3}{7}$

$\frac{3}{7}-\frac{1}{7} x_{1}-\frac{1}{7} \delta_{1}-\frac{3}{7} \delta_{2} \leq 0$

$\frac{3}{7}-\frac{1}{7} x_{1}-\frac{1}{7} \delta_{1}-\frac{3}{7} \delta_{2}+\delta_{3}=0, \delta_{3} \geq 0$

$-\frac{1}{7} x_{1}-\frac{1}{7} \delta_{1}-\frac{3}{7} \delta_{2}+\delta_{3}=-\frac{3}{7}$

إذا القاطع الأكفأ هو القاطع الثاني, إذن نحل السؤال باستخدام القاطع الثاني

لإيجاد القاطع الأكفأ نعوض مقدار النقصان في قيم

الجدول (2-2)

\begin{tabular}{|c|c|c|c|c|c|c|c|}
\hline Basic & $b_{i}$ & $x_{1}$ & $x_{2}$ & $x_{3}$ & $\delta_{1}$ & $\delta_{2}$ & $\delta_{3}$ \\
\hline$x_{3}$ & $6 / 7$ & $-5 / 7$ & 0 & 1 & $2 / 7$ & $-1 / 7$ & 0 \\
\hline$x_{2}$ & $38 / 7$ & $8 / 7$ & 1 & 0 & $1 / 7$ & $3 / 7$ & 0 \\
\hline$Z$ & $132 / 7$ & $23 / 7$ & 1 & 0 & $9 / 7$ & $6 / 7$ & 0 \\
\hline$\delta_{3}$ & $3 / 7$ & $-1 / 7$ & 0 & 0 & $-1 / 7$ & $-3 / 7$ & 1 \\
\hline
\end{tabular}

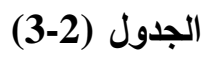

\begin{tabular}{c|c|c|c|c|c|c|c|}
\hline Basic & $b_{i}$ & $x_{1}$ & $x_{2}$ & $x_{3}$ & $\delta_{1}$ & $\delta_{2}$ & $\delta_{3}$ \\
\hline$x_{3}$ & 1 & $-3 / 5$ & 0 & 1 & $2 / 5$ & 0 & -5 \\
\hline$x_{2}$ & 5 & $4 / 5$ & 1 & 0 & $-1 / 5$ & 0 & $3 / 5$ \\
\hline$Z$ & 18 & 3 & 0 & 0 & 1 & 0 & 2 \\
\hline$\delta_{2}$ & 1 & $1 / 3$ & 0 & 0 & $1 / 3$ & 1 & $-7 / 3$ \\
\hline & $x_{1}=0, x_{2}=5, x_{3}=1 \quad, Z=-18$ & & \\
\end{tabular}

استخدام الطريقة الثانية للبرمجة الصحيحة المختلطة الجديدة الجدول (2-1) يمثل الحل الأمثل للمسالة دون الآخذ بعين الاعتبار قيد العدد الصحيح وهو $x_{1}=0, x_{2}=\frac{38}{7}, x_{3}=\frac{6}{7}, Z=\frac{-132}{7}$

قيم أسعار الظل هي

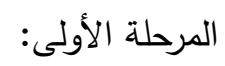
نختار المتغير $x_{3}$ لأنه ذو أعلى كسر أي أن 
$x_{1}=0, x_{2}=5, x_{3}=1, Z_{1}=-18$

$\bar{Z}=Z-Z_{1}=-18 \frac{6}{7}-(-18)=-\frac{6}{7}$

نختبر تحقق القيود

$-(0)-\left(\frac{3}{7}\right)+3\left(\frac{1}{7}\right)=0$

$3(0)-2\left(\frac{3}{7}\right)-\left(\frac{1}{7}\right)=-1$

بما أن القيم ( 1-,0 ) اصغر أو تساوي الصغر فهذا يعني تحقق القيود ومن القيم ( 1-,0 ) يتم اختبار القيد الثاني

$Q_{2}=\left(a_{21}-a_{22}\right)=1$

$\overline{Q_{2}}=\left(c_{1}-c_{2}\right)=5$

لحساب قيم

$$
x_{1}=0, x_{2}=5, x_{3}=1, \mathrm{Z}=-18
$$

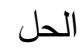

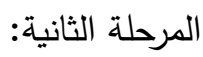

$x_{1}=9, x_{2}=0, x_{3}=0, Z_{2}=18$

$\bar{Z}=Z-Z_{2}=-18 \frac{6}{7}-18=-36 \frac{6}{7}$

1) $-(9)+\left(\frac{-38}{7}\right)+3\left(\frac{-6}{7}\right)=\frac{-119}{7}$

نختبر تحقق القيدين الأول والثاني وعلى النحو الآتي:

2) $3\left(\frac{3}{4}\right)+2\left(\frac{-38}{7}\right)-\left(\frac{-6}{7}\right)=\frac{119}{7}$

إذن لا تحقق القيود

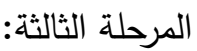

$x_{1}=0, x_{2}=6, x_{3}=0, Z_{3}=-18$

$\bar{Z}=Z-Z_{2}=-18 \frac{6}{7}-(-18)=-\frac{6}{7}$

نختبر تحقق القيود

1) $-(0)+\left(\frac{4}{7}\right)+3\left(\frac{-6}{7}\right)=-2$

2) $3(0)+2\left(\frac{4}{7}\right)-\left(\frac{-6}{7}\right)=2$

إذن لا تحقق القيود

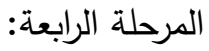

$x_{1}=0, x_{2}=0, x_{3}=6, Z_{4}=-18$

$\bar{Z}=Z-Z_{4}=-18 \frac{6}{7}-(-18)=-\frac{6}{7}$ 
1) $-(0)+\left(\frac{-38}{7}\right)+3\left(\frac{36}{7}\right)=10$

2) $3(0)+2\left(\frac{-38}{7}\right)-\left(\frac{36}{7}\right)=\frac{-112}{7}$

إذن لا تحقق القيود إذن الحل الأمثل هو 18

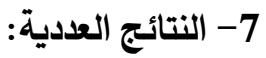

بالاعتماد على خوارزمية قطع المستوي وخوارزمية الزبيدي خوارزميتين NEW NEW2 New الجديدتين ندرج فيما يأتي

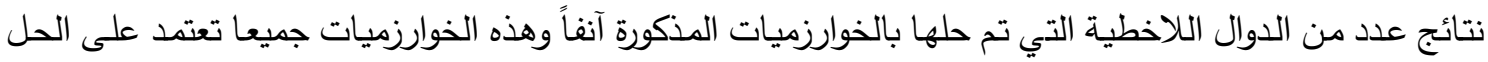
الأمثل.

\begin{tabular}{|c|c|c|c|c|c|}
\hline & PROBLEM & $\begin{array}{l}\text { CUTTING } \\
\text { PLANES }\end{array}$ & Ali H. & NEW 1 & NEW 2 \\
\hline 1. & $\begin{array}{ll}\operatorname{Max} Z=5 x_{1}+2 x_{2} \\
\text { s.t } & 5 x_{1}+4 x_{2} \leq 20 \\
& x_{1}, x_{2} \geq 0\end{array}$ & 20 & 20 & 20 & 20 \\
\hline 2. & $\begin{array}{ll}\operatorname{MaxZ} & =5 x_{1}+8 x_{2} \\
\text { s.t } & x_{1}+x_{2} \leq 6 \\
& 5 x_{1}+9 x_{2} \leq 45 \\
& x_{1}, \quad x_{2} \geq 0\end{array}$ & 40 & 39 & 40 & 40 \\
\hline 3. & $\begin{array}{ll}\operatorname{Max} Z=3 x_{1}+4 x_{2} \\
\text { s.t } \quad 2 x_{1}+x_{2} \leq 6 \\
& 2 x_{1}+3 x_{2} \leq 9 \\
& x_{1}, x_{2} \geq 0\end{array}$ & 12 & 10 & 12 & 12 \\
\hline 4. & $\begin{array}{cc}\operatorname{Max} Z=5 x_{1}+4 x_{2} \\
\text { s.t } & x_{1}+x_{2} \leq 5 \\
& 10 x_{1}+6 x_{2} \leq 45 \\
& x_{1}, \quad x_{2} \geq 0\end{array}$ & 23 & 23 & 23 & 23 \\
\hline 5. & $\begin{aligned} \text { MinZ }= & 2 x_{1}-3 x_{2}-3 x_{3} \\
\text { s.t } \quad & -x_{1}+x_{2}+3 x_{3} \leq 8 \\
& 3 x_{1}+2 x_{2}-3 x_{3} \leq 18 \\
& x_{1}, x_{2}, x_{3} \geq 0\end{aligned}$ & -18 & -18 & -18 & -18 \\
\hline 6. & $\begin{array}{ll}\operatorname{MaxZ}= & 4 x_{1}+5 x_{2}+7 x_{3} \\
\text { s.t } \quad & 3 x_{1}+2 x_{2}+3 x_{3} \leq 20 \\
& 3 x_{1}+5 x_{2}+7 x_{3} \leq 30 \\
& 2 x_{1}+3 x_{2}+4 x_{3} \leq 25 \\
& x_{1}, x_{2}, x_{3} \geq 0\end{array}$ & 33 & 33 & 33 & 33 \\
\hline
\end{tabular}




\section{المصادر}

الزبيدي, د.علي خليل, (2000) " الطريقة المقترحة لحل مسائل البرمجة الخطية ", المؤتمر العلمي الثالث

عشر للجمعية العراقية للعلوم الإحصائية, مجلة العلوم الإحصائية الصفحة (334-348).

احمد, د. خالد حسين, (2000) " بحوث العمليات أسس ومبادئ ", مطبعة وزارة التعليم العالي والبحث

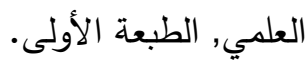

صالح, د. بشير محمد, صالح, محمد واجد محمد, (2005) " تحسينات الطريقة الفردية المتوازنة ", مجلة

$$
\text { الرافدين لعلوم الحاسبات والرياضيات, المجلد (2), العدد (2), الصفحة (67-72). }
$$

فتوحي, نوار نجم عبد الله, (2005) " طريقـة تهجينية لقطع المستويات مـع طريقة التقرعات في مسائل

$$
\text { البرمجة الصحيحة " رسالة ماجستير , غير منشورة. }
$$

[5] AdAm, N. LetchFord AND Andrea Lodi, (2001) "Primal Cutting Plane ALGORITHM REVISITED " LANCASTER LA 14 YW, V, K, PP.

[6] R. S. Grafinkel and G. L. Nemhauser, (2003) "INTEger Programming", MATH3902 OPERATION RESEARCH II, CHAPTER 1, PP. (1-5).

[7] RALPH, E. GOMORY, (1973) "AN ALL-INTEGER PROGRAMMING ALGORITHM", RAND REPORT, R. M. 25797, NEW YORK CHAPTER 3, PP. (46-S66).

[8] Salkim, Harrey M., (1975) "Integer programming Addision - wesley publishing Co., Inc., Reading, Mass chapter 2, pp. 34. 